Int J Microcirc 1996;16:327-328

\title{
Subject Index Vol. 16, 1996
}

Acidosis 180 Adenosine 308 Albumin concentration Ill Angiogenesis 8, 82, 227, 232, 302 Angioma 82 Angiotensin II 302 Anti-angiogenesis 165 Architecture 1 Area vasculosa 165 LArginine 221 Arterial autograft 240 Arteriolar network 232

- $\quad$ spasm 98

Arterioles 308

Autoregression analysis 64

Basal lamina 291

Basic fibroblast growth factor 8

Blister suction technique Ill

Blood flow regulation, cutaneous 259

Brain 89

Capillaries 137

Capillaroscopy 16, 23, 271, 313

Capillary blood pressure analysis, newly developed software 129

- velocity 137

density 23,271

diameter 74 -, glass 187

structure 284

Cell-free (plasma) layer, marginal 187 Cerebral autoregulation 277

blood flow 277

cortex 143

Chorioallantoic membrane 82 Chronic heart failure 137

- $\quad$ venous insufficiency 320

Cobra venom factor 89

Co-culture 105

Colloid osmotic pressure Ill Coronary microcirculation 170 Cremaster muscle 98 Critical

limb ischemia 57

- $\quad$ red cell transit time 118

Dermofluorography 195

Dextran 187

Diabetes mellitus 313

Diamide 187

Digital image processing 195

Endothelial cell 105

- $\quad$ gaps 291

Endothelin 250

receptor subtype 250 Endotoxin 89 Erythrocyte aggregation 187

deformation (deformability) 187 Esterase staining 147 Extracellular matrix components 302 
Femorodistal bypass 284 Fibroblast growth factors 308 Fluorescein angiography 195 Flux motion 64 Fourier analysis 64

Ginkgo biloba extract (EGb 761) 98 GinkgolideB 98

Hamster cheek pouch 308 Hemoconcentration 266 Hemodynamics 129 Heparin 8

Hepatic microcirculation 250 Hepatocyte 105 Heterogeneity 1,271 Human skeletal muscle

284 Hypercarbia 180 Hyperoxia 143 Hyperventilation 266 Hypoxia 291

Iloprost 284

Immunosuppression 147 Interferon- $\alpha 165$ Intimal hyperplasia 240 Intravital microscopy 74

Isch(a)emia 180,284 Ischemia/reperfusion 89, 170

Laser Doppler 64

- flowmetry 221,277

- flux 16,259

- fluxmetry 124,313

Left ventricular ejection fraction 137 Leg edema Ill Leukocyte(s) 89, 170 Long-term followup 57 Lower limb atherosclerosis Ill Lung 89

Mast cells 82

Maturation 232

Medical compression stockings 320

Mesenteric vessels 227

KAIUiEH

E-Mail karger@,karger.ch Fax+ 41613061234 http://www. karger. ch

(C) 1997 S. KargerAG, Basel

327

Mesentery, rabbit 187 Microangiopathy 259 Microcirculation 1, 57, 89, 137 -, skin 16

Microelectrode 143, 180 Microvascular growth 232 Microvasculature 147 Molecular mass 8

Monocrystalline antimony 180 Multiple indicator dilution method 291 Muscles 1

Myeloperoxidase assay 89

$\mathrm{N} \omega$-nitro-L-arginine 277 Neovascularization 308 Neutrophil 89 Nitric oxide 98

Oxygen 143

diffusion barrier 118

extraction rate 118

tension 74

Pancreatic autodigestion 221

- $\quad$ perfusion 221

Pentoxifylline 170

Perfusion pressure, reduction 74

Peripheral arterial occlusive disease 64

Permeability 105

Permeability-surface area product $291 \mathrm{pH}$ distribution 180 Platelet-activating factor 98

Postural feedback system 259 Prostaglandin Ei 57

Protein concentration Ill Pulse pressure 129

Quantification 8, 227

Rat 170

Raynaud's phenomenon 16

Reactive hyperemia 137 Relaxin 227 Reperfusion 284 
- $\quad$ injury 170

Ritanserin 143

Serotonin 143 Skin 271

- $\quad$ microcirculation 313

Sodium fluorescein 266

Sponge implant 302

Stress test, local cold 16

Subcutaneous interstitial fluid Ill

Sympathectomy 195

Taurocholate pancreatitis 221 tcp $<3 / 457$

Test, distant cooling 124 -, inspiratory gasp 124 Thromboxane 98 Transcapillary transport 266 Tuberculin reaction 118 Tumor necrosis factor- $\alpha 147$

Ultrastructure 291

Vasoconstriction 259, 308 Vasodila(ta)tion 74, 308 Vasomotor reflex, skin 124

Vasoproliferative processes 82 Venivasomotor response 259 Venolymphatic drainage 320

Venous incompetence, chronic 259

- $\quad$ ulcer 259

Venuloarteriolar reflex 259

Wick technique Ill

328

Subject Index Vol. 16, 1996 